Research Article

\title{
Prediction and Aerodynamic Analysis of Interior Noise and Wind Drag Generated by the Outside Rear-View Mirror for Commercial Vehicles
}

\author{
Binfei Hu, ${ }^{1}$ Zengjun Lu, ${ }^{2}$ Qiming Cui, ${ }^{3}$ Rongjiang Tang $\mathbb{D}^{1,2}{ }^{1,2}$ Ze Feng, ${ }^{2}$ and Daokun $\mathrm{Bi}^{2}$ \\ ${ }^{1}$ School of Mechanical and Electrical Engineering, Guilin University of Electronic Technology, Guilin 541004, China \\ ${ }^{2}$ Dongfeng Liuzhou Motor Co., Ltd., Liuzhou 545005, China \\ ${ }^{3}$ Beiben Trucks Group Co., Ltd., Beijing 100000, China
}

Correspondence should be addressed to Rongjiang Tang; tangrongjiang@guet.edu.cn

Received 9 July 2020; Revised 14 August 2020; Accepted 20 August 2020; Published 14 September 2020

Academic Editor: Hong-Jun Zhu

Copyright (c) 2020 Binfei Hu et al. This is an open access article distributed under the Creative Commons Attribution License, which permits unrestricted use, distribution, and reproduction in any medium, provided the original work is properly cited.

The outside rear-view mirror (OSRVM) is installed on the vehicle's surface, which causes unwanted aerodynamic noise and wind drag during driving. It is important to use simulation methods to predict the performance of aerodynamic noise and wind drag of commercial vehicles due to the OSRVM. Considering the wind drag of the OSRVM, a combinational simulation strategy is employed to calculate external flow and interior acoustic fields of commercial vehicles, respectively. The flow field is computed a priori with an incompressible flow solver. The acoustic field was then computed based on the information extracted from the CFD solver. To obtain the interior noise level at the driver's ears, a vibroacoustic model is used to calculate the response of the window glass structure and interior cavities, where the unsteady aerodynamic pressure loading on the two side windows' surface is treated as the acoustic source field. The paper provides flow field and acoustic simulations for three OSRVM configuration models. The results are compared to data obtained in road sliding test measurement on the commercial vehicle. The accuracy of the hybrid simulation method is proved, and the comparative analyses verify that the OSRVM B model dramatically reduces the interior noise and wind drag of commercial vehicles.

\section{Introduction}

The importance of commercial vehicles as medium and long-distance transportation tools has been increasing with the rapid development of the logistics industry. Noise, vibration, and harshness (NVH) are now significant in commercial vehicles, and automotive companies are interested in levels of NVH as a component of the development of comfortable vehicles [1]. The commercial vehicle test proves that when the driving speed is greater than $90 \mathrm{~m} / \mathrm{s}$, the impact of aerodynamic noise on the interior vehicle is most prominent in broadband frequency noise, directly affecting interior sound quality and driver comfort.

Meanwhile, aerodynamic noise and wind drag are important parameters for the aerodynamic performance of commercial vehicles. In general, the outside rear-view mirror is also known to increase the total amount of wind drag by 2 7\% [2]. The selection of the outside rear-view (or side-view) mirror (OSRVM) and design modification are needed for the reduction of aerodynamic noise and wind drag. The OSRVM is installed on the vehicle's surface to guarantee the driver sees the areas behind and to the sides of the vehicle. During driving, the high-speed airflow flows through the outer surface of the vehicle are more prominent, resulting in a serious separation of the airflow flowing through the mirror area, and the turbulent pressure generated pulsates against the window, causing strong aerodynamic noise inside the vehicle. It is of great significance to study the transient flow field and sound field propagation for the drag and noise reduction of the whole vehicle.

At present, researchers have made considerable research on the unsteady flow field and sound field of automobile 
rear-view mirrors. Kucukcoskun et al. [3] reported the distribution of sound source on the surface of the rear-view mirror. The computational fluid dynamics (CFD) combined with the acoustic finite element method (FEM) is used to calculate the distribution of the sound field outside the vehicle. A method for predicting the noise outside the vehicle is obtained by comparing the wind tunnel experiment. However, it did not predict the aerodynamic noise inside the cab. Schell and Cotoni [4] combined CFD with the acoustic wave equation to propose the air-vibration-acoustic simulation method to predict the sound pressure level in the cab, and the sound pressure level near the driver's ear was obtained by incompressible unsteady flow excitation. However, it did not consider the effects of different rear-view mirrors on the interior noise and the effects of wind drag for the whole vehicle.

Chu et al. [5] compared the aerodynamic characteristics of the OSRVM with the inner ducts and original mirror models. The modified mirror models can enhance the aerodynamic flow and noise around the mirror. This study did not achieve the goal of reducing the sound pressure level in the cab. Mutnuri et al [6] combined CFD with statistical energy analysis (SEA) to evaluate the design of the rear-view mirror aerodynamic noise. The conclusion was obtained that the aerodynamic noise of the rear-view mirror mounted on the glass is larger than the aerodynamic noise of the rearview mirror mounted on the door from quantitative and qualitative analysis, which indicated the installation position of the rear-view mirror has a great influence on the interior noise and helped to guide the design of the rear-view mirror installation. Gu et al. [7] verified the feasibility of the largeeddy simulation method contrasted the test result and used this method to calculate the buffeting noise data of the car. The interior acoustics were analyzed combined with the weak vibration radiation of the window with the partially open window. However, they did not study the effect of fully enclosed windows on the interior noise. Iida $\mathrm{K}$ et al. and Yamade $Y$ et al. $[8,9]$ employed the finite element method to calculate the hydrodynamic pressure fluctuation of the vehicle surface as the unsteady load and predicted the internal sound field of the test vehicle by solving the Helmholtz acoustic propagation equation. The contribution from the external acoustical field to the interior noise was estimated based on the acoustic analogy and was confirmed to be negligibly small compared with that from the hydrodynamic loading in the present case. Moreover, aerodynamic sources and noise generation generated by the rear-view mirror have been investigated by numerous experimental and simulation measures [10-13].

Some typical prediction strategies for the interior noise to commercial vehicles are needed in order to reduce the aerodynamic noise. Besides, more studies have employed aerodynamic optimization techniques to reduce the strength of the intrinsic shedding vortex and suppress the generation of the aerodynamic noise by changing the geometric shape of the rear-view mirror, such as its bracket position, the housing modeling and height and size, the elimination of sharp edges on the housing surface, and increasing the microwind guide structure $[14,15]$. However, there are limitations to reducing the geometry of the side mirror for aerodynamic noise in commercial vehicles, and these measures apply merely to passenger vehicles.

Very little study has been undertaken to investigate the aerodynamic noise of the rear-view mirror under considering the effect of wind drag in commercial vehicles, and the rear-view mirror has a considerable effect on the wind drag of commercial vehicles. There is little research on reducing the aerodynamic noise and wind drag by studying the modeling factors of the rear-view mirror for commercial vehicles. Accordingly, the object of this study is to consider the aerodynamic flow field and sound field radiation by external shape design of three OSRVMs. The commercial program STAR-CCM+ was used to analyze the wind drag coefficient variation according to the shape of the OSRVM. Based on computational fluid dynamics (CFD) analysis, this study investigates aerodynamic flow characteristics at three major points around the mirror housing and sound pressure levels in the commercial vehicles' cab.

\section{Numerical Calculation of the Outside Rear-View Mirror with the Cab and Vehicle}

2.1. DES Governing Equations. The wind drag of the external flow field and the steady-state calculation of the cab model are based on the realizable $k-\varepsilon$ model. Realizable $k-\varepsilon$ which is a standard $k-\varepsilon$-improved extended model performs well in solving the flow field in the rear-view mirror area. Transport equation for $k$ turbulent kinetic energy is given [16] as

$$
\frac{\partial(\rho k)}{\partial t}+\frac{\partial\left(\rho k u_{i}\right)}{\partial x_{i}}=\frac{\partial}{\partial x_{j}}\left[\left(\mu+\frac{\mu_{t}}{\sigma_{k}}\right) \frac{\partial k}{\partial x_{j}}\right]+G_{k}-\rho \varepsilon .
$$

Transport equation for $\varepsilon$ turbulent dissipation ratio is

$$
\frac{\partial(\rho \varepsilon)}{\partial t}+\frac{\partial\left(\rho \varepsilon u_{i}\right)}{\partial x_{i}}=\frac{\partial}{\partial t}\left[\left(\mu+\frac{\mu_{t}}{\sigma_{\varepsilon}}\right) \frac{\partial \varepsilon}{\partial x_{j}}\right]+\rho C_{1} E \varepsilon-\rho C_{2} \frac{\varepsilon^{2}}{k+\sqrt{v \varepsilon}}
$$

where $\rho$ is the air density, $u_{i}$ is the velocity component in the $x_{i}$ direction, $\mu$ and $\mu_{t}$ are laminar viscous coefficient and turbulent viscous coefficient, $\sigma_{k}$ and $\sigma_{\varepsilon}$ are the Prandtl number corresponding to the turbulent kinetic energy $k$ and dissipation rate $\mathcal{E}$ (the values are 1.0 and 1.2 ), $G_{k}$ is the turbulent kinetic energy $k$ produced by the average velocity gradient, $C_{1}$ and $C_{2}$ are experience constants (the values are 1.42 and 1.68), and $E$ is the user defined number and provided by STAR-CCM+. The model ensures the continuity of turbulence in Reynolds pressure. It has a more accurate prediction of cylindrical spoiler and has good convergence and accuracy.

Detached eddy simulation (DES) combines the advantages of Reynolds-averaged Navier-Stokes (RANS) and large eddy simulation (LES). It is solved by RANS in the near-wall area, which makes the calculation in the boundary layer small. The far wall area is solved by LES, which better simulates the state of large-scale separation turbulent flow. Combining them organically improves computational 
efficiency and accuracy. The dissipation rate of the kinetic energy equation in DES based on the realizable $k-\varepsilon$ model [17] is

$$
\left\{\begin{aligned}
Y_{k} & =\frac{\rho k^{(3 / 2)}}{d}, \\
d & =\min \left[\frac{k^{(3 / 2)}}{\varepsilon}, C_{\mathrm{DES}} \Delta\right],
\end{aligned}\right.
$$

where $C_{\mathrm{DES}}$ is for the calibration of the DES model, where $\Delta=\max \left(\Delta_{x}, \Delta_{y}, \Delta_{z}\right)$. When $k^{(3 / 2) / \varepsilon}<C_{\mathrm{DES}} \Delta, d=k^{(3 / 2)} / \varepsilon$, realizable $k-\varepsilon$ model plays the role of RANS. Conversely, when $d=C_{\mathrm{DES}} \Delta$, the equation shows the characteristics of the LES subgrid-scale.

2.2. Computational Model. A simple commercial vehicle cab model is chosen to predict the aerodynamic noise for saving computing resources and calculation time. As shown in Figure 1, the real model is extracted from the whole commercial vehicle, which was represented as a type of commercial vehicle. The left and right rear-view mirrors, the right lower-view mirror, the sun visor, and the front lower mirror are externally highlighted to ensure that they are in accordance with the experimental result. The length, width, and height of the commercial vehicle cab model are $2.0 \mathrm{~m}$, $2.5 \mathrm{~m}$, and $2.1 \mathrm{~m}$, respectively. The OSVRMs are mounted on a simplified commercial vehicle cab. OSRVM A shown in Figure 1(a) is the original reference mode, which is commonly used in commercial vehicles for observing the aerodynamic flow. It has rear-view mirror cover of $15 \mathrm{~cm}$ and can be seen in its cross section. OSRVM B displayed in Figure $1(\mathrm{~b})$ is the dislodging rear-mirror cover. The airflow passes from the windward side to the trailing edge of the mirror housing, whose rear-view mirror cover has vital influence for wind drag and aerodynamic noise. While comparing OSRVM C, its windward side was changed that the edge of regression on the windward side was moved toward the left. OSVRM A with the simplified cab is shown in Figure 1(d). The real commercial vehicle cab is shown in Figure 1(e). It is the same as the geometric model. The commercial vehicle model with the trailer is seen in Figure 1(f) and is used to study the aerodynamic drag performance analysis under full-cargo conditions.

2.3. Numerical Simulation Condition Setting. To improve the accuracy of the CFD analysis results and reduce the number of calculations, the surface mesh size of the vehicle is set to $10-150 \mathrm{~mm}$ for the aerodynamic analyses. The OSRVM, side window, and A-pillar surface mesh are approximately $2 \mathrm{~mm}$. The volume mesh is generated based on the created surface mesh. The calculated domains are all four-layer encryption, and the encryption area of the rear-view mirror area is $2 \mathrm{~mm}$ (simulations can be obtained within $2500 \mathrm{~Hz}$ sound pressure level). In the DES analysis, the required Y+ wall value is less than 5 . The DES boundary layer is shown in Table 1 based on the first boundary layer thickness and the total thickness formula to obtain the corresponding boundary layer structure and determine a reasonable boundary layer and grid growth rate. The number of trim meshes used in the calculations reached 65 million. The simulation adopts the high-speed working condition of the CV at $100 \mathrm{~km} / \mathrm{h}$, and the inlet is the turbulence intensity (calculated as $I=0.06$ ) and the characteristic length, which is taken as the length of the rear-view mirror. The pressure and velocity subrelaxation factors of the separation flow are set to 0.3 and 0.7 , respectively. Table 2 describes the boundary conditions for the total computational domain. A cuboid domain was used to perform the CFD simulations, as illustrated in Figure 2, which considers the airflow rate and field, where the dimensions of the computational domain are $20 \mathrm{~L} \times 5 \mathrm{~W} \times 6 \mathrm{H}$.

The transient calculations use the steady-state results as the initial values to solve the incompressible flow. The result of steady-state computational convergence is shown in Figure 3. Before the transient calculations, the side windows are defined as the sound source surface, and the CGNS sound source data code is the output. The acoustic analogue method is then used to solve the sound pressure level of the window surface. Realizable $k-\varepsilon$ is applied to calculate the steady-state behaviour, and the parameters used for the calculations are presented in Table 3. The DES model is applied to calculate the transient-state behaviour, and the set for the transient-state solver is given in Table 4. The total time duration is set to $0.5 \mathrm{~s}$, and the time step for the calculation is $0.0002 \mathrm{~s}$. As the computational solution gradually tends to be stable, the results between 0.2 and $0.5 \mathrm{~s}$ are selected for the analysis.

2.4. Aerodynamic Drag Performance Analysis. As there are few wind tunnels suitable for commercial vehicles and they have high associated costs, the calculations for the aerodynamics of commercial vehicles with three OSRVMs are from the CFD method based on variations of the drag coefficient or the rate of velocity and transient pressure. Any vehicles that travel through a fluid experience drag forces in the direction of the flow due to the pressure and shear forces that act on the surface of an object. The aerodynamic drag coefficient can be expressed as

$$
C_{d}=\frac{F_{d}}{(1 / 2) \rho A v^{2}} .
$$

Here, $F_{d}$ is the aerodynamic drag force $(N), \rho$ is the fluid density, $A$ is the characteristic frontal area of the commercial vehicle with the rear-view mirror $\left(\mathrm{m}^{2}\right)$, and $v$ is the velocity of the operational vehicle $(\mathrm{m} / \mathrm{s})$.

All models are simulated under the commercial vehicle driving speed of $100 \mathrm{~km} / \mathrm{h}(27.8 \mathrm{~m} / \mathrm{s})$. The wind drag coefficient for the three rear-view mirrors is shown in Table 5. OSRVM B cancels the aerofoil of the rear-view mirror relative to OSRVM A. The table shows that the aerofoil of the rear-view mirror does not influence the aerodynamic drag for the entire commercial vehicle model. From OSRVM C, the windward side of the rear-view mirror is blunted, and the corresponding drag coefficient is higher than that for OSRVM A. $Z=C d \times A$ is the power consumption factor, 


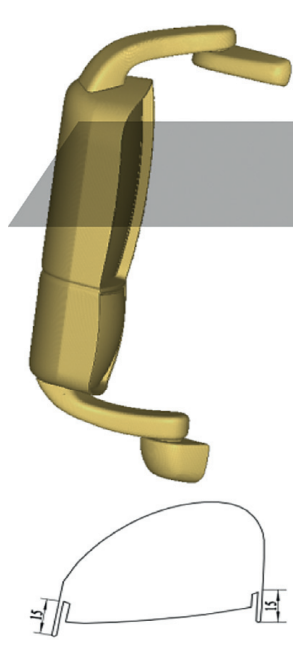

(a)

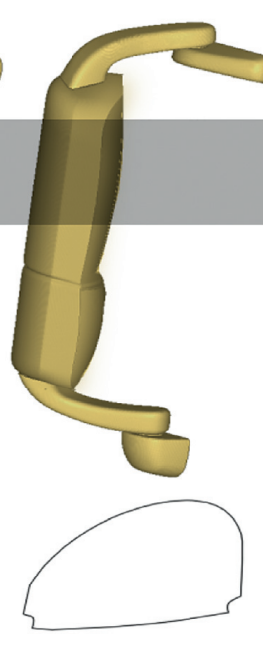

(b)

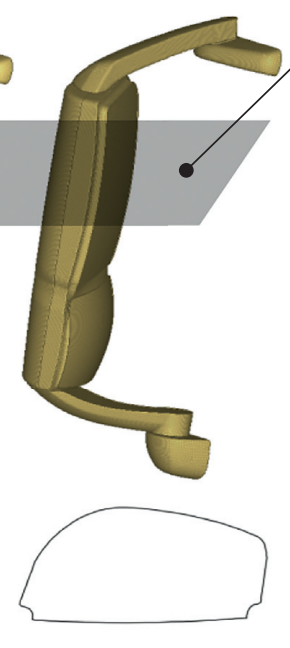

(c)

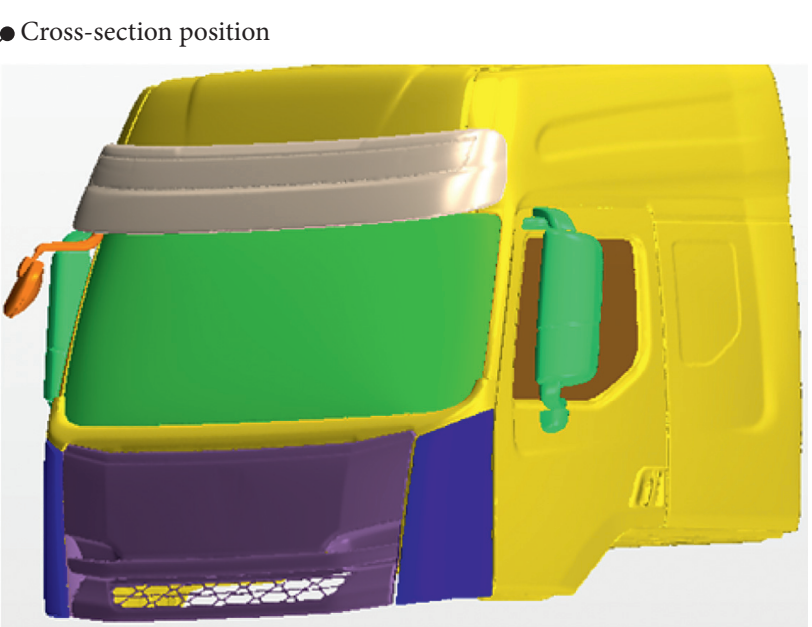

(d)

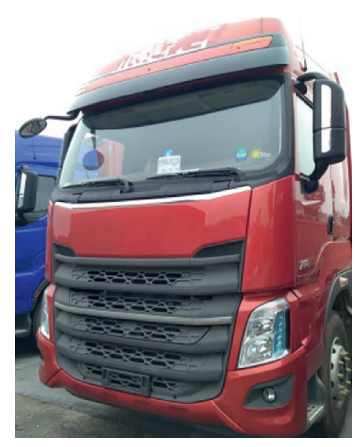

(e)

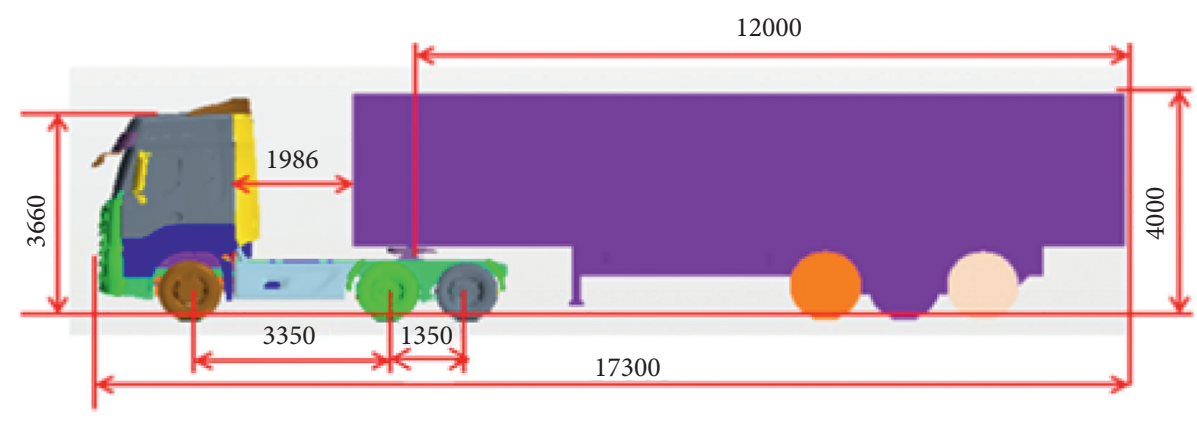

(f)

FIGURE 1: OSRVM cab mode and commercial vehicle cab: (a) OSRVM A with its cross section; (b) OSRVM B with its cross section; (c) OSRVM C with its cross section; (d) cab mode with the characteristic object; (e) the commercial vehicle with a special figure in real status; (f) the commercial vehicle with the trailer.

TABle 1: DES boundary layer.

\begin{tabular}{lc}
\hline Item & Data \\
\hline First layer thickness $(\mathrm{mm})$ & 0.2 \\
Rate of rise & 1.12 \\
Total layer number & 10 \\
Total thickness $(\mathrm{mm})$ & 3 \\
\hline
\end{tabular}

TABle 2: Set of the boundary condition.

\begin{tabular}{lc}
\hline Boundary of the domain & Set \\
\hline Inlet & Velocity inlet: $v_{x}=100 \mathrm{~km} / h$ \\
Outlet & Pressure outlet \\
Rear-view mirror; cab; floor & Wall (no slip) \\
Top; side face & Wall (free slip) \\
\hline
\end{tabular}

which is used to illustrate the aerodynamic drag related to the front projection area of the car cab. The table indicates that the rear-view mirror with passivation at the windward surface does not appreciably reduce the wind resistance comparing the values of mirror B and mirror A. Compared with cancelling of the rear-view mirror, the drag coefficient is the highest, up to 0.6349 , although the corresponding orthographic projection area is the smallest, and the power

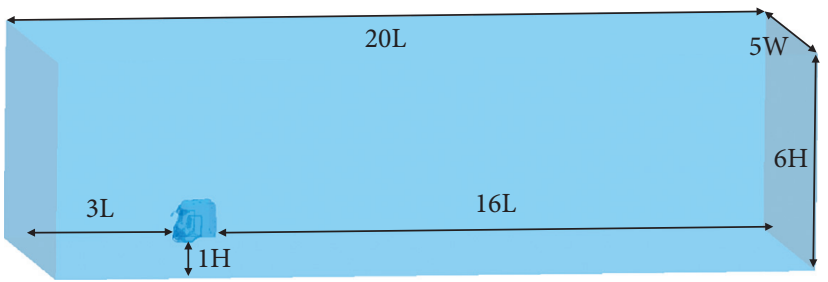

FIgURE 2: Computational domain around the commercial vehicle $\mathrm{cab}$ and its dimension.

consumption factor is indeed the largest. Therefore, the rearview mirror helps reduce the wind drag and improve the aerodynamic performance of the commercial vehicles.

The aerodynamic drag coefficient $\left(C_{d}\right)$ for the four cases in the commercial vehicles with a trailer (Figure 4 ) changes for the rear-view mirrors and trailer. Figures 4(a)-4(c) illustrate the vehicle aerodynamic drag development curve for the three rear-view mirror cases of vehicles with trailers. Rear-view mirrors A, B, and C relative to the original car resistance development curve do not significantly differ. For example, when the rear-view mirror resistance slightly increased, no obvious difference was observed in $C_{d}$. The aerodynamic drag for rear-view mirrors of different shapes 


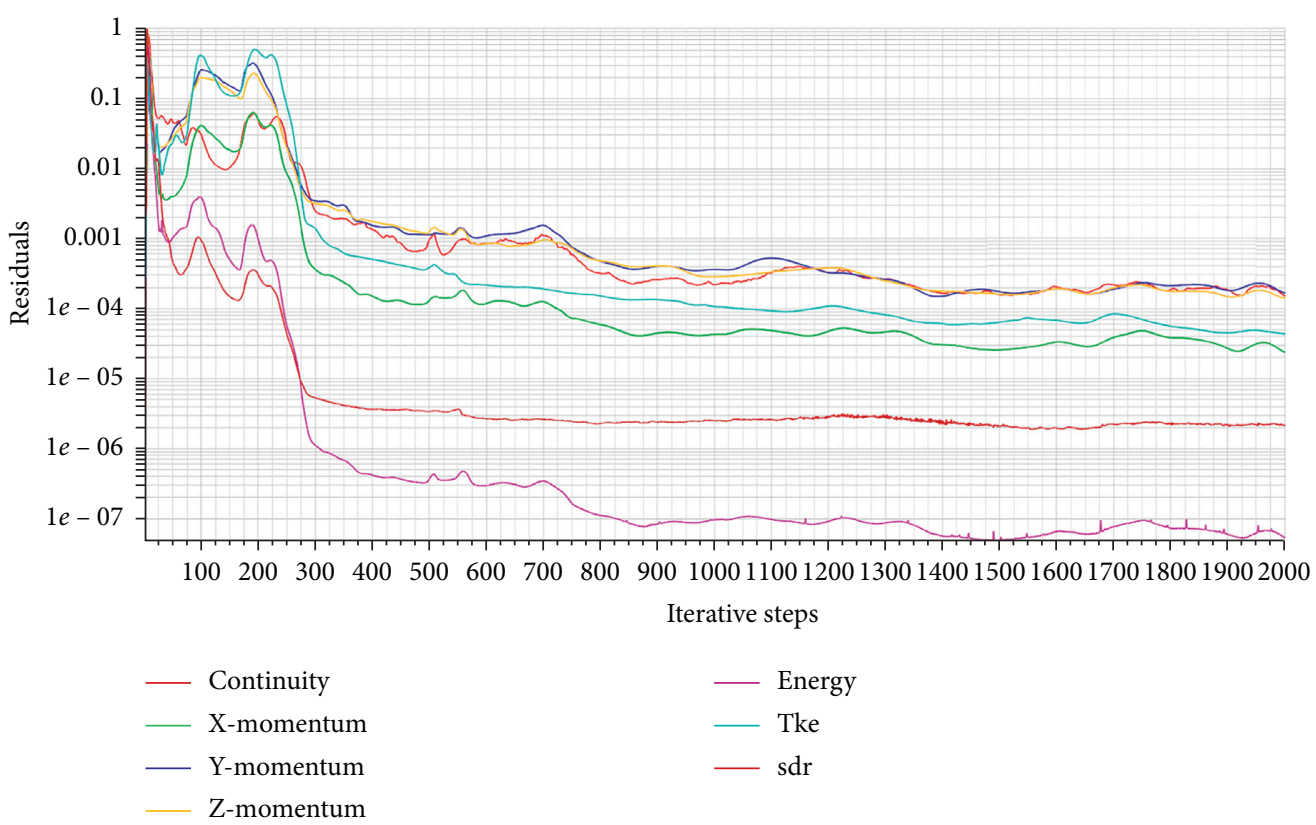

FIgURE 3: Residual convergence curve of the realizable $k-\varepsilon$ model.

TABLE 3: Set of steady parameters.

\begin{tabular}{lc}
\hline Item & Set \\
\hline Air property & Ideal gas \\
Pressure-velocity coupling & Simple \\
Pressure spatial discretization & Second order \\
Momentum spatial discretization & Second-order upwind \\
Turbulent kinetic energy & Second-order upwind \\
\hline
\end{tabular}

TABLE 4: Set of the transient parameter.

\begin{tabular}{lr}
\hline Item & Set \\
\hline Pressure-velocity coupling & Piso \\
Momentum spatial discretization & Bounded central differencing \\
Density spatial discretization & Second-order upwind \\
\hline
\end{tabular}

TABLE 5: Comparison of the aerodynamic performance of four rear-view mirrors.

\begin{tabular}{lcccc}
\hline OSRVM plan & Orthographic projected area $\left(A / m^{2}\right)$ & Wind drag coefficient $\left(C_{d}\right)$ & $C d \times A$ & $Z$ discrepancy \\
\hline OSRVM A & 10.227 & 0.5809 & 5.9409 & Base \\
OSRVM B & 10.227 & 0.5809 & 5.9409 & $0 \%$ \\
OSRVM C & 10.204 & 0.5891 & 6.0112 & $1.2 \%$ \\
NO OSRVM & 9.912 & 0.6349 & 6.2931 & $5.9 \%$ \\
\hline
\end{tabular}

also shows negligible effects on commercial vehicles with a trailer. In contrast with the case without a rear-view mirror, $C_{d}$ showed obvious changes in the rear-view mirror and trailer regions with increased $C_{d}$. This is consistent with the results in Table 5. A preliminary assessment of the presence of the rear-view mirror can improve the flow field behind the mirror and reduce the aerodynamic drag.
The steady-state velocity flow field with or without the rear-view mirror is shown in Figure 5. The elliptical dashed circle in the A pillar leads to the separation of air flow on the side. The rear-view mirror can guide the air flow back and reduce the eddy current in the side area. The rear-view mirror can reduce the negative pressure area of the side of the cab and avoid the airflow directly shocking the trailer. 


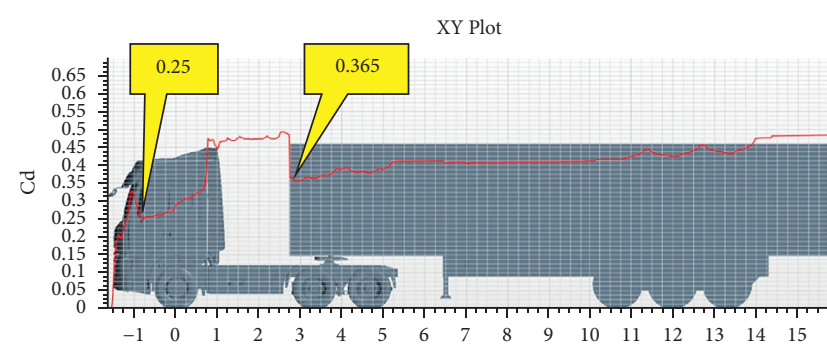

$\mathrm{m}$

(a)

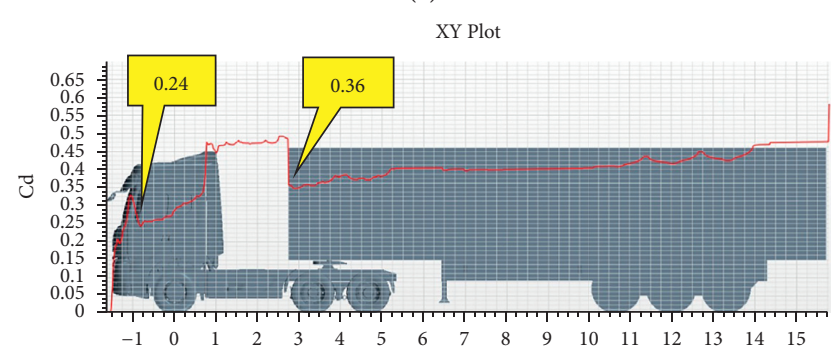

$\mathrm{m}$

(c)

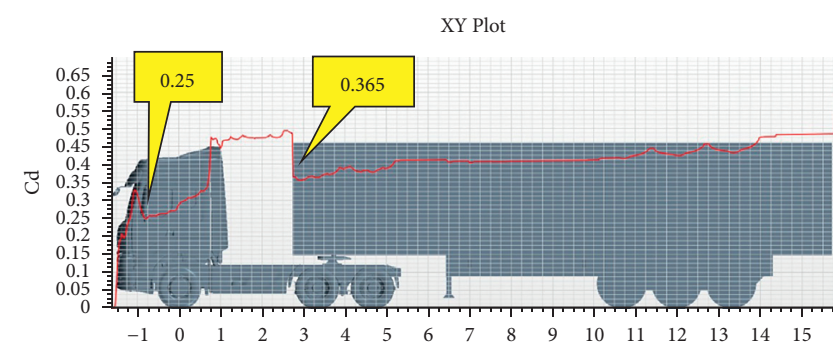

m

(b)

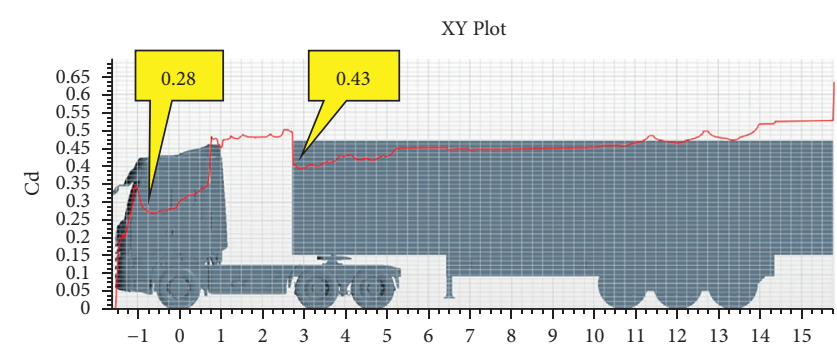

m

(d)

Figure 4: Wind drag development curve of four status commercial vehicles. (a) OSRVM A. (b) OSRVM B. (c) OSRVM C. (d) NO OSRVM.

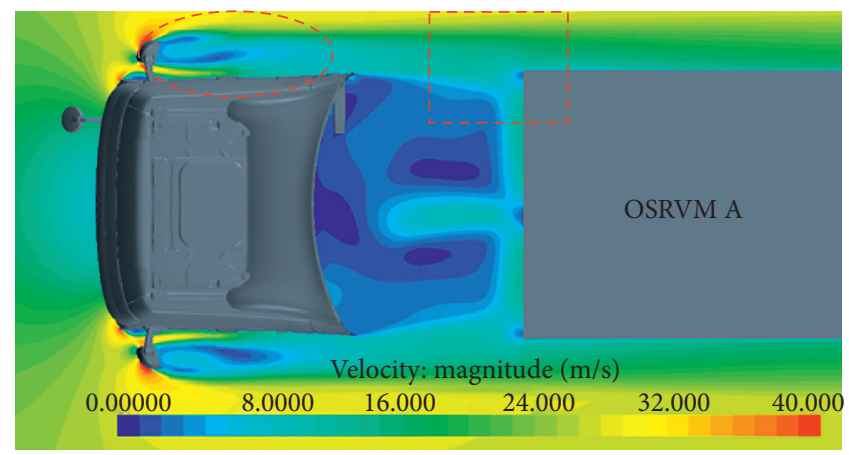

(a)

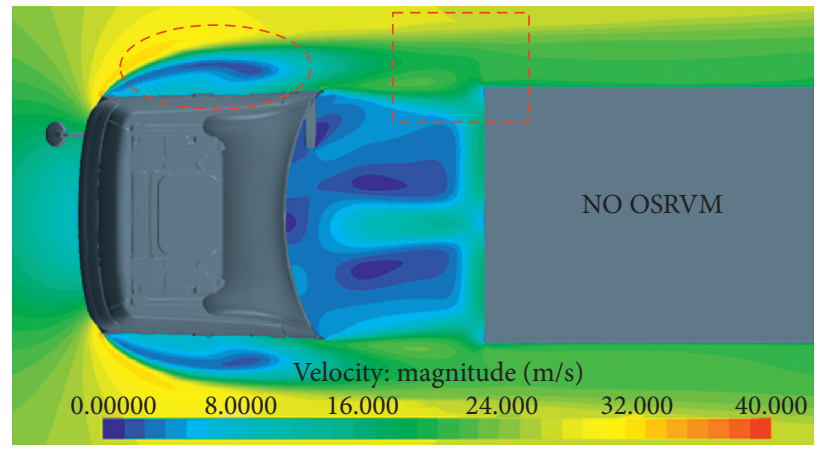

(b)

FiguRE 5: Flow velocity field contour of commercial vehicles with the trailer: (a) with the rear-view mirror; (b) without the rear-view mirror.

For commercial vehicles with the trailer, the wind drag without the rear-view mirror status is higher.

2.5. Unsteady Flow Analysis. The Z-section is created to study the airflow field and aerodynamic characteristics of the rear-view mirror tail region, which is parallel to the XOY plane, and is shown in Figure 6 (top). Figures 6(a)-6(d) show the transient velocity contour for the three OSVRMs and the NO OSRVM, respectively. The velocity magnitudes at the side window, windward side, and trailing of the mirror are displayed in the graphs, which symbolize three representative velocity directions and magnitude. The airflow velocity of OSRVM A is relatively larger than the other model around the windward side and side window. In particular, the airflow velocity increases significantly at the windward side to $50 \mathrm{~m} / \mathrm{s}$ at a vehicle speed of $30 \mathrm{~m} / \mathrm{s}$. However, OSVRM C shows a lower airflow velocity in the place of side window and windward side, which is a moderate change in the area around the rear-view mirror. Compared with NO OSRVM, there is little airflow on rushes at the side window, and the airflow velocity is significantly reduced. Therefore, the NO OSRVM case is a promising way to reduce the influence of airflow shocks against the side window, that is, the highpressure fluctuations around the mirror housing that propagate the vibrations to the window are accordingly reduced [18].

Figures $7(a)-7(d)$ show the transient pressure contours for the four models at typical driving speeds. Overall, airflow through the mirror has a pressure gradient from the 


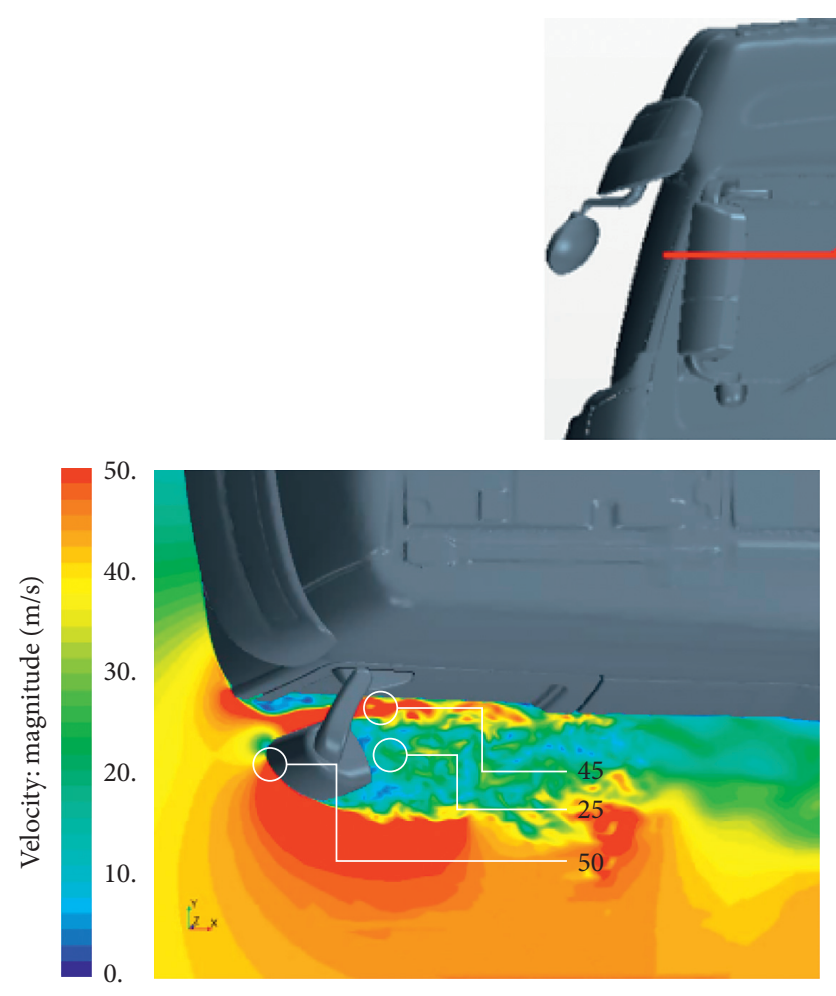

(a)

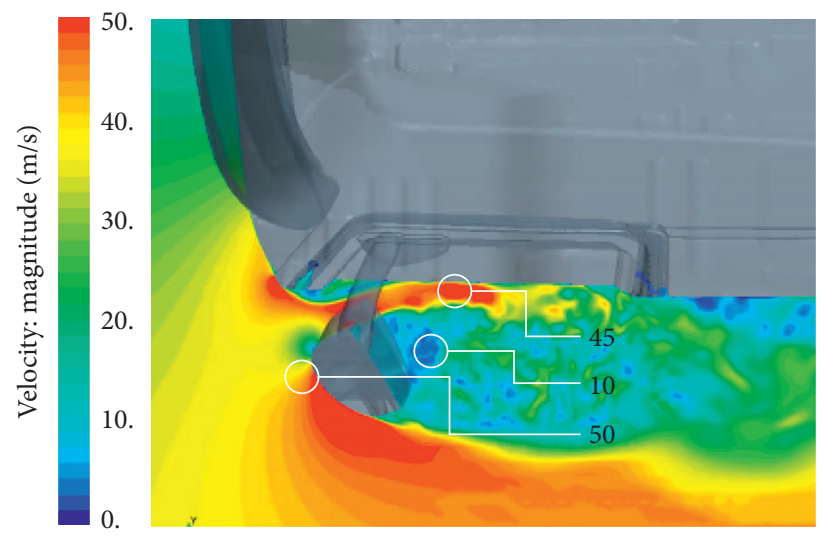

(c)

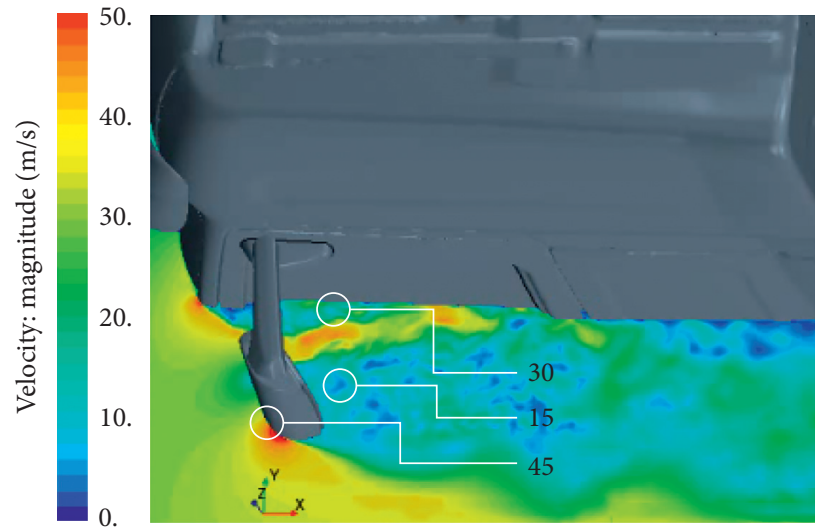

(b)

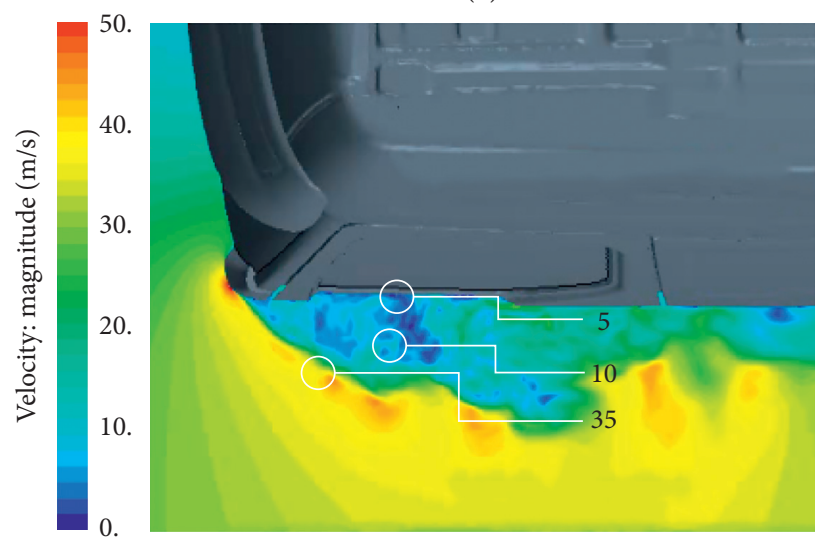

(d)

FIgURE 6: The transient velocity cloud for the three OSRVM and NO SORVM cases with three labeled velocities. (a) OSVRM A. (b) OSRVM B. (c) OSRVM C. (d) NO OSRVM.

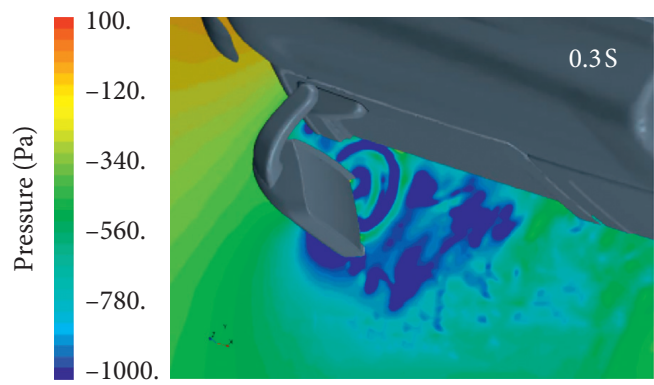

$-1000$

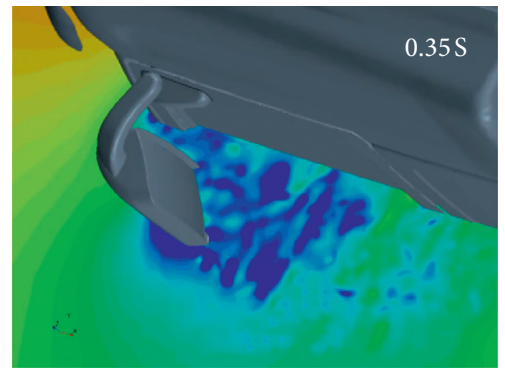

(a)

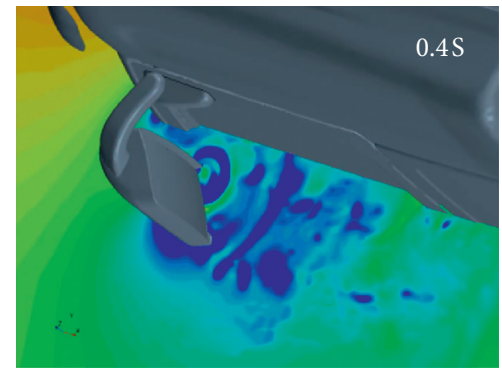

$0.4 \mathrm{~S}$

Figure 7: Continued. 

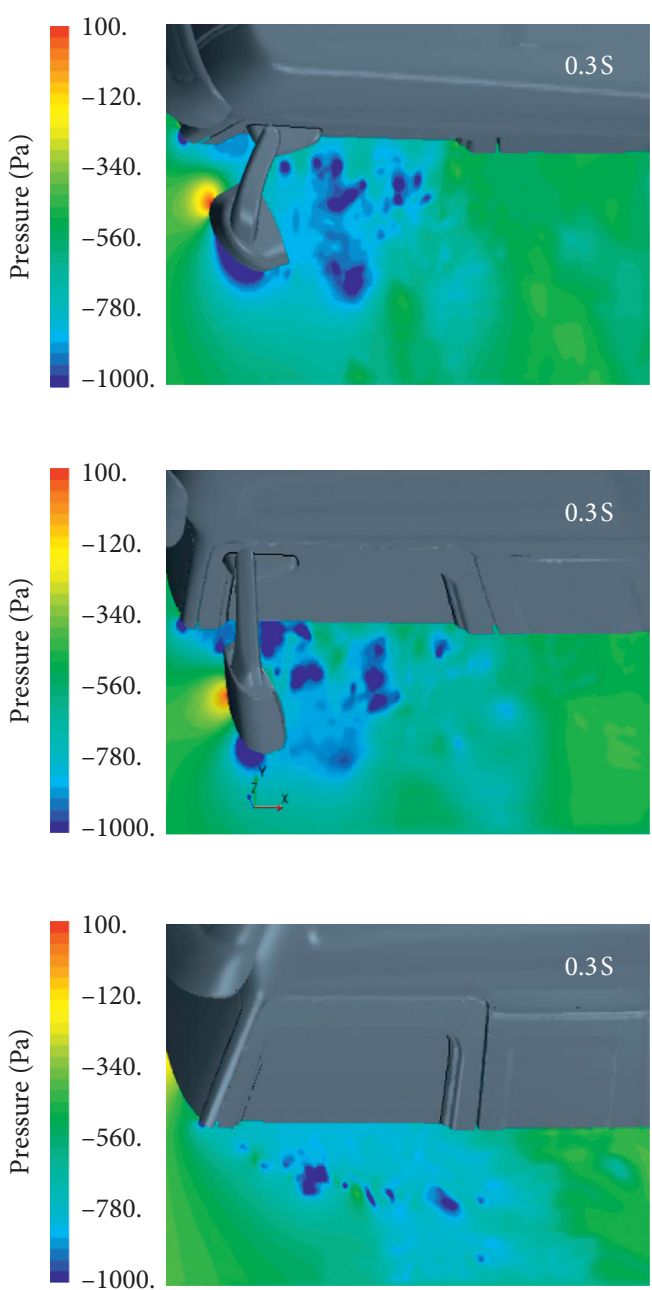

$-1000$

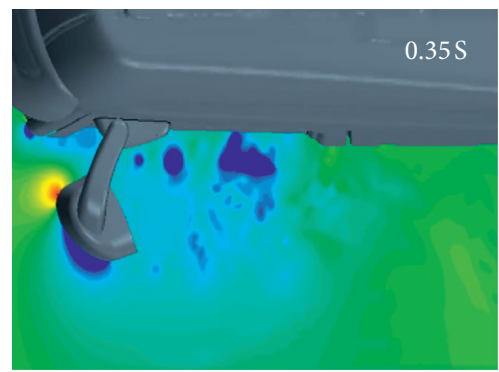

(b)

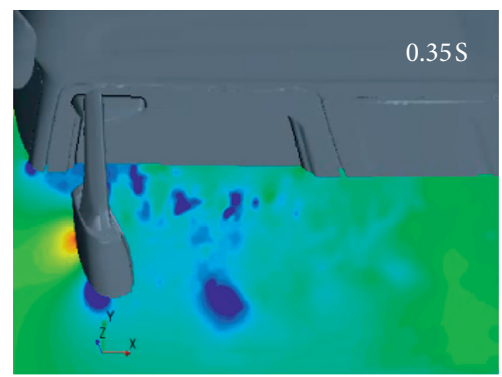

(c)
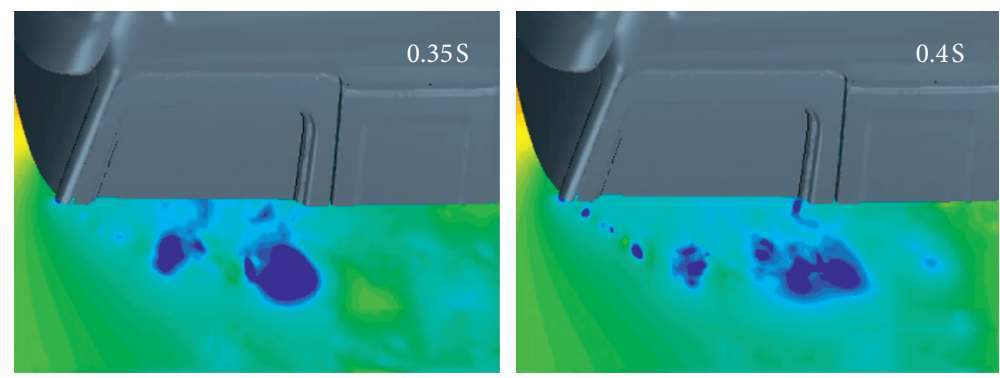

(d)

FIgURe 7: The transient pressure contour at different times for (a) OSRVM, (b) OSRVM B, (c) OSRVM C, and (d) NO OSRVM.

negative to the positive pressure zones and forms a pressure vortex with a periodic direction of rotation that is opposite to its rotation $[19,20]$. The various airflow vortices significantly affect the flow field outside the vehicle, which can reduce or increase the aerodynamic drag and noise for commercial vehicles. OSVRM A shows that a circular pressure contour appears at the junction of the lens and the frame and periodically changes over time. The transient pressure flow field in OSRVM A is more disordered compared to OSRVM B, which forms a large low-pressure vortex in the rear-view mirror tailing region. The airflow vortices of OSRVM C are relatively small. The first three rear-view mirror modes cause each eddy current to impinge on the window glass and generate a vortex that becomes squeezed, enlarged, and broken in the rear-view mirror tailing area. There is a strong pressure pulsation in the wake field, which is accompanied by the generation of aerodynamic noise [21-23]. A small low-pressure vortex is formed in the rear-view mirror tailing region for the $\mathrm{NO}$ OSRVM case, and the low-pressure vortex develops backwards with time and gradually evolves into a large lowpressure vortex. The formed low-pressure cavity in the space increases the air resistance of the vehicle, which is not conducive to reduce the drag of the commercial vehicles.

\section{Dynamic Analysis of the Window Structure}

The vibration of the window can be excited from a fluctuating pressure, which affects the flow field in two ways: the boundary condition of the flow field and the elastic waves caused by the vibrations. The influence of the vibrations on the fluid is ignored, and those caused by other excitations are omitted as the magnitude of the displacement caused negligible vibrations and a Mach number which is less than 1.

3.1. Governing Equations. The differential equations of motion for a damped $n$-degree-of-freedom system can be expressed in the matrix form as

$$
M \ddot{\delta}+C \dot{\delta}+K \delta=P(t)
$$

where $M$ is the mass matrix, $K$ is the stiffness matrix, $C$ is the damping matrix, and $P$ is the vector of generalized nonconservative forces. 
For preliminary application of modal analysis, we must solve the eigenvalue problem, which is given by

$$
K \varphi_{r}=\omega_{r}^{2} M \varphi_{r} .
$$

Equation (6) has the solutions $\omega_{r}^{2}$ and $\varphi_{r}(r=1,2,3 \cdots n)$, where $\omega_{r}$ are the natural frequencies and $\varphi_{r}$ are the natural modes (modal vectors). The eigenvalues and eigenvectors can be arranged in the $n \times n$ matrix form:

$$
\left\{\begin{array}{l}
\Omega=\operatorname{diag}\left[\omega_{1}^{2} \omega_{2}^{2} \omega_{3}^{2} \cdots \omega_{n}^{2}\right], \\
\Phi=\left[\varphi_{1} \varphi_{2} \cdots \varphi_{n}\right]^{T},
\end{array}\right.
$$

where $\Phi$ is the modal matrix. Moreover, it is convenient to normalize the modal matrix so as to satisfy the orthonormality relations:

$$
\left\{\begin{array}{r}
\Phi^{T} M \Phi=I \\
\Phi^{T} K \Phi=\Omega .
\end{array}\right.
$$

The damping matrix can be expressed as a linear combination of the mass and stiffness matrices of the form

$$
C=\alpha M+\beta K,
$$

where $\alpha$ and $\beta$ are known scalar constants. Multiplying equation (9) on the left by $\Phi^{T}$ and on the right by $\Phi$ and considering equation (8) give the diagonal matrix:

$$
\Phi^{T} C \Phi=\alpha I+\beta \Omega \text {. }
$$

The solution of equation (5) can be expressed as a linear combination of the modal vectors as

$$
\delta(t)=\sum_{r=1}^{n} q_{r}(t) \varphi_{r}=\Phi q(t),
$$

where $q_{r}(t)$ represents the modal coordinates and $q(t)$ is the corresponding vector.

Next, equation (11) is substituted into equation (5), and the results are left-multiplied by $\Phi^{T}$. Then, considering equations (8) and (10) gives

$$
\ddot{q}(t)+(\alpha I+\beta \Omega) \dot{q}(t)+\Omega q(t)=\Phi^{T} P(t)=N(t) .
$$

This has the notation of

$$
\alpha+\beta \omega_{r}^{2}=2 \zeta_{r} \omega_{r}(r=1,2,3 \cdots n),
$$

where $\zeta_{r}(r=1,2,3 \cdots n)$ are modal viscous damping factors. Equation (12) can be rewritten in the form of the independent modal:

$$
\ddot{q}_{r}(t)+2 \zeta_{r} \omega_{r} \dot{q}_{r}(t)+\omega_{r}^{2} q_{r}(t)=N_{r}(t)(r=1,2,3 \cdots n),
$$

where $N_{r}(t)$ are modal forces having the form given as $N_{r}(t)=\varphi_{r}^{T} P(t)(r=1,2,3 \cdots n)$.

3.2. Computational Details. HyperMesh software is applied to calculate the glass-constrained modes of the side windows, and HyperWork shell 63 is chosen as the element type.
The elastic connection unit was employed to simulate the window seal knot, and glass is used as the medium for data transmission between solids and gases. The acoustic harmonic FEM in the LMS Virtual.Lab uses interpolation to map the CFD time-domain data onto a structural grid for the glass. The modal superposition method is adopted as it has a rapid calculation speed, a high solution precision, and a relatively small required disk space. There are 100 modals, and the sound pressure level within $2500 \mathrm{~Hz}$ is calculated using Fourier transform with a calculation step of $5 \mathrm{~Hz}$. Young's modulus, Poison's ratio, the tempered glass density and thickness are $7.2 E 10 \mathrm{~Pa}, 0.21,2520 \mathrm{~kg} / \mathrm{m}^{3}$, and $3.5 \mathrm{~mm}$, respectively.

Figure 8 describes the change processes for displacements at the two side windows. The order of displacement magnitude is between $10 E-3$ and $10 E-8 \mathrm{~m}$, and the displacement in the $y$ direction is greater than for the other directions. As shown in Figure 8, the displacement magnitude at the two side windows forms peaks and valleys, which increase in number at higher frequencies. There are separated flows and vortices around the two side windows, and the turbulent kinetic energy and pressure around the left front side-window are greater than those around the right front side-window, which is sometimes opposite. Thus, the displacement gradients along for left and right side-windows are considered.

\section{Acoustic Simulation}

4.1. Governing Equations. The acoustic pressure is governed by the classical wave equation, expressed by

$$
\nabla^{2} p^{\prime}-\frac{1}{c^{2}} \frac{\partial^{2} p^{\prime}}{\partial t^{2}}=-\rho_{0} \frac{\partial p^{\prime}}{\partial t},
$$

where $\nabla^{2}$ is the Laplacian operator, $p^{\prime}$ is the acoustic pressure, $c$ is the speed of sound in the acoustic medium, and $q \prime$ is the added mass caused from unit volume acceleration. When only the harmonic steady-state case is considered, the classical wave equation reduces to the Helmholtz equation as

$$
\nabla^{2} p-k^{2} p=-j \rho_{0} \omega^{\prime} q
$$

where $k=\omega / / c$ is the acoustic wave number, $\omega /=2 \pi f$ is the angular frequency, $f$ is the frequency $(\mathrm{Hz}), j=\sqrt{-1}$, $p^{\prime}=p \cdot \exp (j \omega t)$, and $q^{\prime}=q \cdot \exp (j \omega t)$. The solution for equation (16) satisfies the boundary condition as follows:

$$
n \nabla p=\omega^{\prime 2} \rho_{0} n U_{s},
$$

where $n$ denotes the unit normal pointing into the boundary and $U_{s}$ denotes the displacement vector. The solution can be derived by the indirect boundary element method, given as

$$
p=A_{\sigma}^{T} \cdot \sigma+A_{\mu}^{T} \cdot \mu,
$$

where $\sigma$ is the single-layer potential vector, $\mu$ is the doublelayer potential vector, and $A_{\sigma}^{T}$ and $A_{\mu}^{T}$ are coefficient vectors corresponding to $\sigma$ and $\mu$, respectively. 


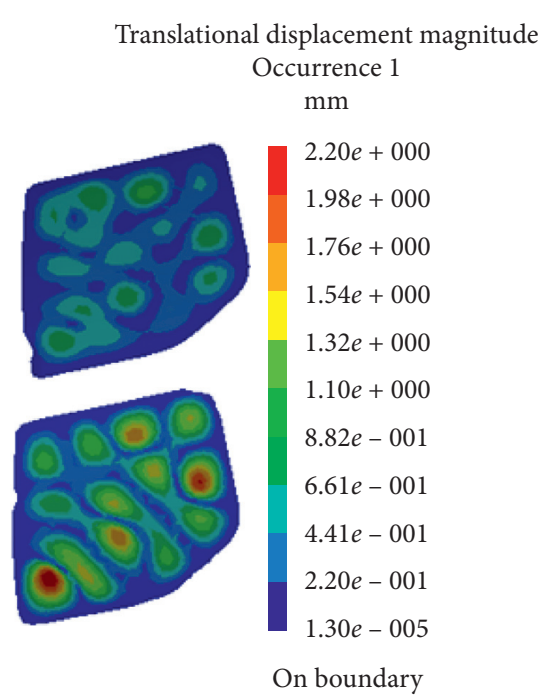

$20 \mathrm{~Hz}$

Translational displacement magnitude Occurrence 8

$\mathrm{mm}$

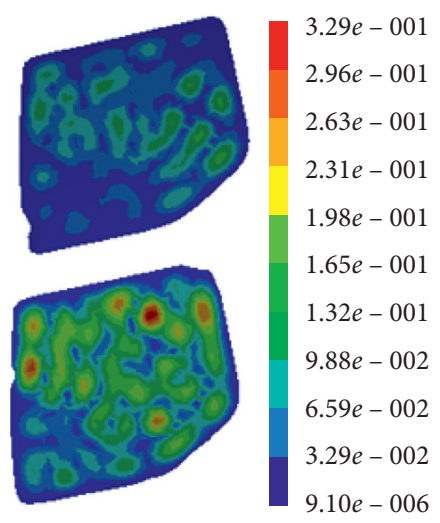

On boundary

$40 \mathrm{~Hz}$

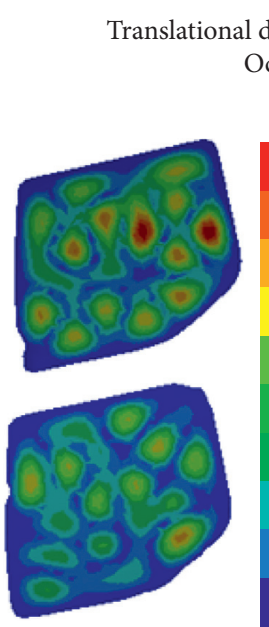

Occurrence 3
mm

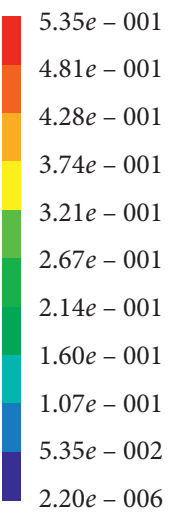

On boundary

$25 \mathrm{~Hz}$

Translational displacement magnitude Occurrence 12

$\mathrm{mm}$
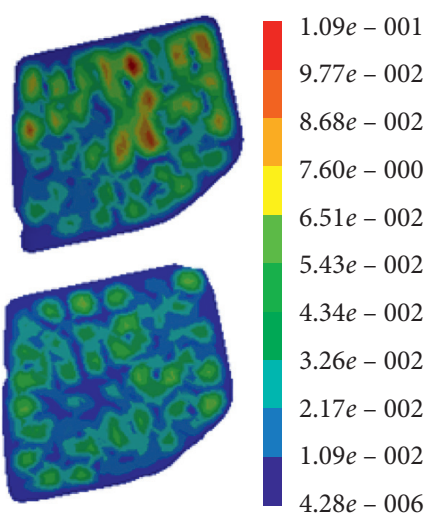

On boundary

$50 \mathrm{~Hz}$
Translational displacement magnitude Occurrence 5

$\mathrm{mm}$

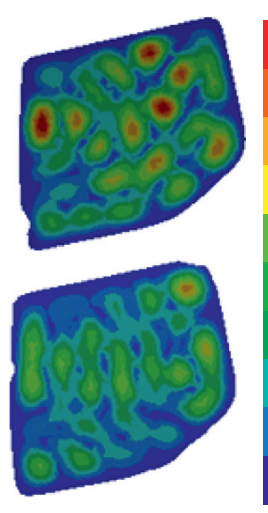

$3.42 e-001$
$3.08 e-001$
$2.73 e-001$
$2.39 e-001$
$2.05 e-001$
$1.71 e-001$
$1.37 e-001$
$1.03 e-001$
$6.83 e-002$
$3.42 e-002$
$9.65 e-006$

On boundary

$31.5 \mathrm{~Hz}$

Translational displacement magnitude Occurrence 16 $\mathrm{mm}$

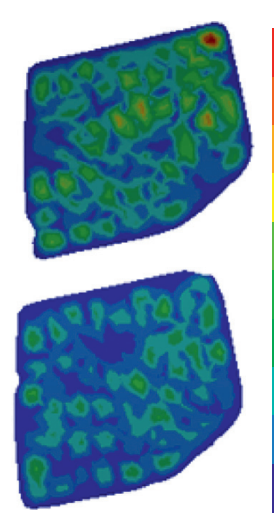

$9.49 e-002$

$8.54 e-002$

$7.59 e-002$

$6.64 e-002$

$5.70 e-002$

$4.75 e-002$

$3.80 e-002$

$2.85 e-002$

$1.90 e-002$

$9.50 e-003$

$3.47 e-006$

On boundary

$63 \mathrm{~Hz}$

FIgURE 8: Responses of the two side windows of the fluctuating pressure.

The sound pressure at an arbitrary point, says, in the field can be expressed as the summation of all the panels' noise contributions at the point:

$$
p_{s}=\sum_{i=1}^{n} p_{i, s}
$$

where $p_{s}$ is the total sound pressure at point $s$ and $p_{i, s}$ is the sound pressure contribution at point $s$ from panel $i$, which is given by

$$
p_{i, s}=\sum_{j=1}^{m} p_{i, j}
$$

where $p_{i, j}$ denotes the sound pressure for panel $i$ from modal j.

For illustrating the meaning of $p_{i, j}$ more distinctly, the normalized factor is introduced as follows:

$$
R p=\frac{p_{s}^{*}}{\left|p_{s}\right|^{2}}
$$

where $p_{s}^{*}$ is the complex conjugate; then, the modal participation factor of modal $j$ from panel $i$ can be written as

$$
p_{c}=\operatorname{Real}\left(p_{i, j}^{*} \cdot R p\right),
$$

where Real denotes the real part of the complex. Positive $p_{c}$ indicates that the sound pressure $p_{s}$ increases with the elevation of the response of panel $i$ from modal $j$. Negative $p_{c}$ means that the sound pressure $p_{s}$ decreases as the response of panel $i$ from modal $j$ increases.

4.2. Results and Discussion. The boundary conditions of the acoustic finite element method include the following: the two side-window elements are set as the displacement boundary condition, the seat elements are removed, the roof 

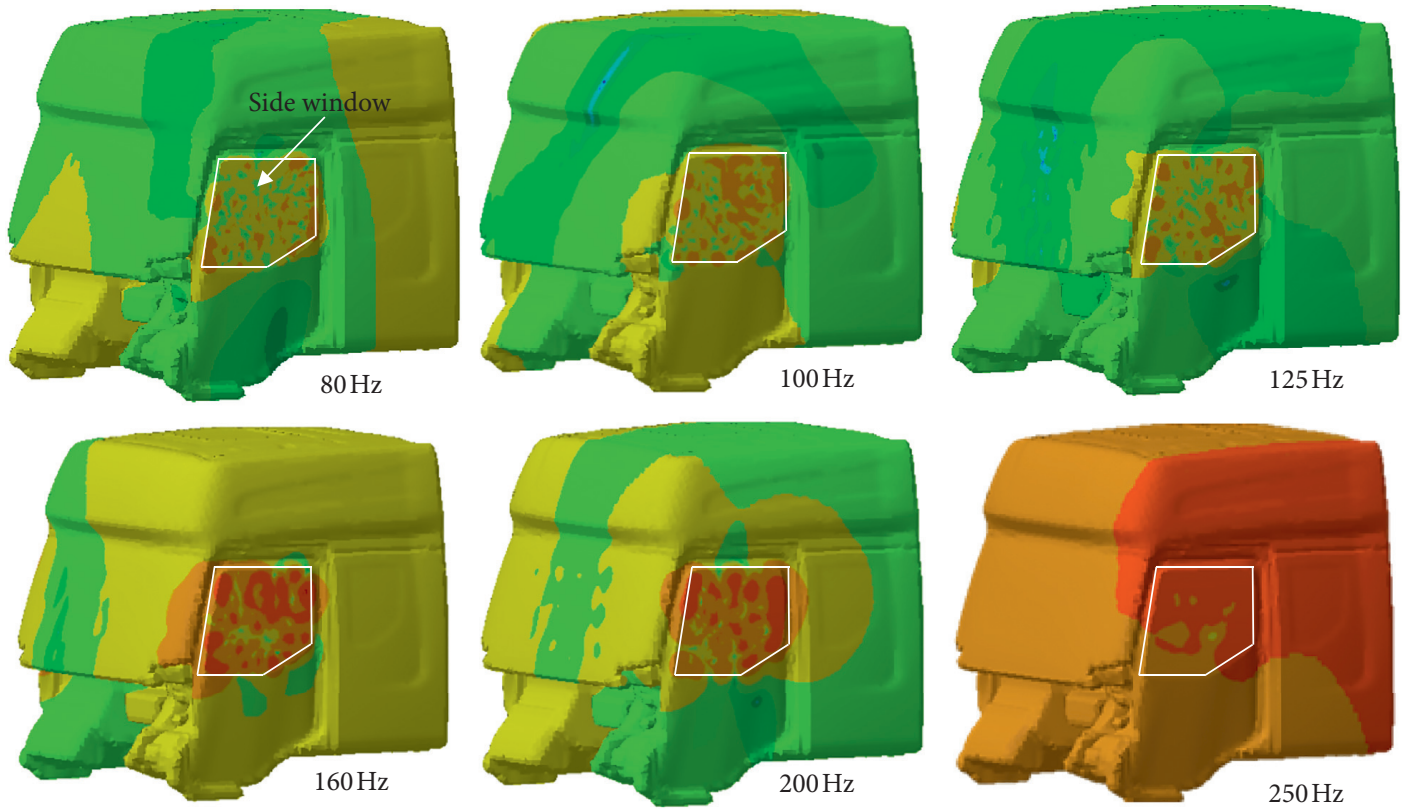

FIgure 9: The distribution of the OSRVM A sound pressure level in the cab cavity.

elements are set as the automatically matched layer boundary condition (no reflection boundary), and the car door and interior trim were dismantled, which were substituted by the outermost surface of a car [24, 25]. Other elements are set as the simple panel, which is also set as the automatically matched layer boundary condition.

The cab sound cavity is set as the computational field and is created to analyze the distribution of the sound pressure level (SPL) on the surface of the cab. As shown in Figure 9, the SPL contour gradually decreases from the side window to the other locations of the passenger cab compartment at frequencies of $80,100,125 \mathrm{~Hz}$, etc. At some selected frequencies, the cloud maps indicate that the maximum SPL of the noise is distributed around the side window, and the SPL gradually increases with frequency in the range of $20-400 \mathrm{~Hz}$. The SPL then gradually reduces when over $400 \mathrm{~Hz}$, but the sound pressure area near the side window is still the largest. Therefore, the side-window area of the rearview mirror is the primary source of noise while driving and is also the main noise source for the passenger.

The two vertical and horizontal planes through the points of the driver's left and right ear were set as the computational field. As shown in Figure 10, the SPL contour of the acoustic space inside the cab can be seen such that the influence of the aerodynamic noise on the interior of the vehicle is mainly concentrated on the place around the driver's left ear. The noise in the driver's head area is greater than other spaces when the frequency is $125 \mathrm{~Hz}$ and $250 \mathrm{~Hz}$.

The SPL contour images in Figure 11 for different OSRVMs at 400 and $500 \mathrm{~Hz}$ show that the SPL distributions around the side-window surface are higher than the other regions at the same frequency. Comparing the SPL contours for OSRVM A and B, the side-window surface emerges as the largest SPL region that exceeds $90 \mathrm{~dB}$. This suggests that the rear-view mirror cover between the lens and the frame reduces the aerodynamic noise to some extent. This is limited to reducing the noise near the window as the overall change is not large. The SPL of OSRVM C is lower than for $\mathrm{B}$, which means that the windward side of the rear-view mirror influences the SPL of the rear-view mirror area. Appropriately changing the parameters for both mirrors reduces the aerodynamic noise that propagates to the vehicle. Therefore, a proper rearview mirror cross section is beneficial to reduce the aerodynamic noise and drag. The SPL distributions of the sound cavity without the mirror are the smallest. Combined with the transient speed and pressure contour, the NO OSRVM reduces the turbulent pressure pulsation at the mirror area and reduces the aerodynamic noise, which is derived primarily from the pressure fluctuations of the A-pillar airflow separation [14, 23, 26-28]. Compared with the sound pressure distribution at $500 \mathrm{~Hz}$, the SPL tends to decrease with frequency, and the SPL of the window surface becomes increasingly important.

Figure 12 shows the SPL distribution of the interior vehicle acoustic space for the $1 / 3$ octave centre frequency of the three OSRVMs and the NO OSRVM case. The 400 and $500 \mathrm{~Hz}$ SPL contours show that OSRVM A has the highest SPL in the cab. This indicates that the influence of OSRVM A on the wind noise inside the vehicle is large, while OSRVM B and $\mathrm{C}$ reduce the noise inside the vehicle. However, the SPL for the NO OSRVM in the car is the lowest, which is $45 \mathrm{~dB}$ near the left ear of the driver. It is seen that the gap between the lens and the frame influences the aerodynamic noise inside the vehicle. The windward side of the rear-view mirror affects the SPL in the car and is lower in the centre of the 
SPL (dB) A
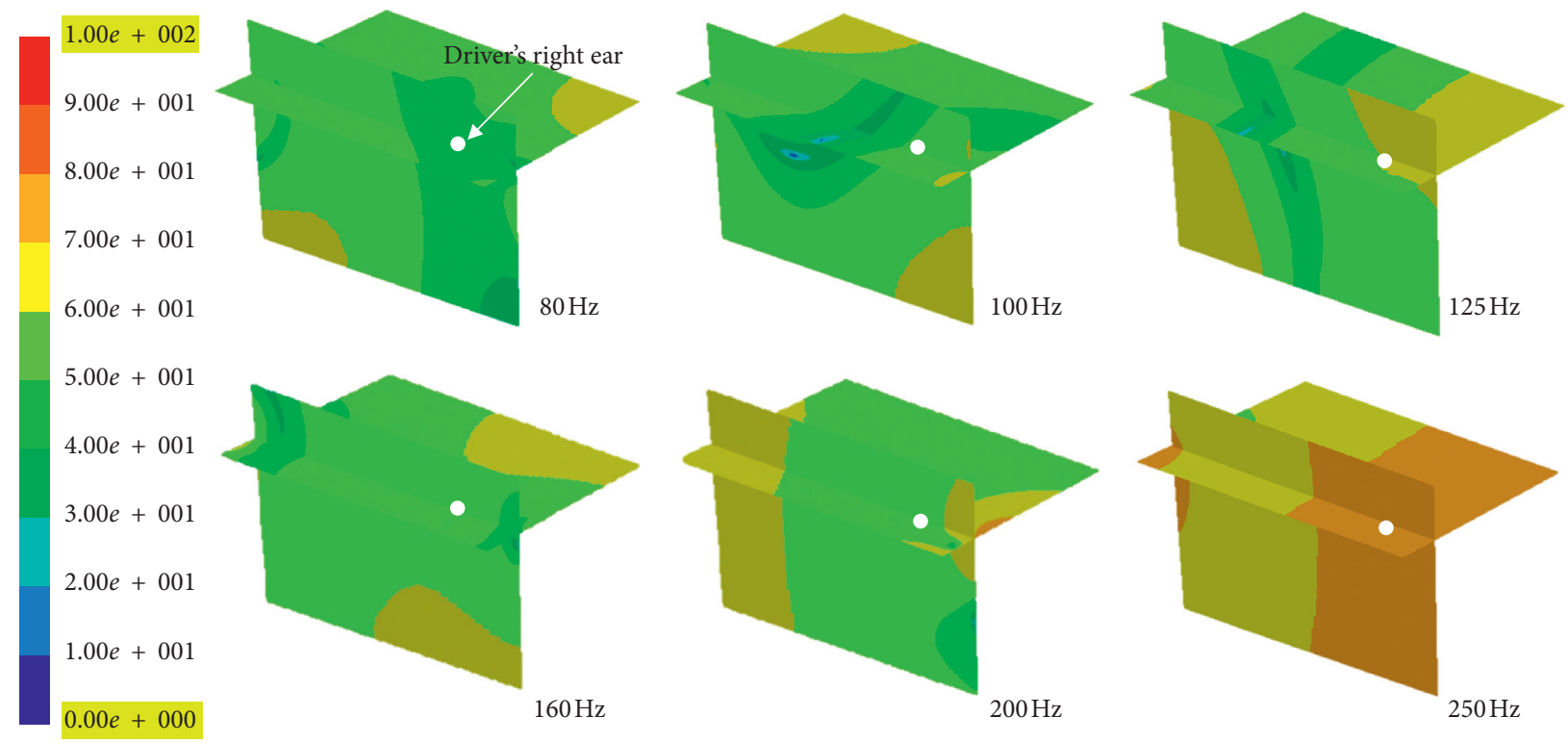

FIgURE 10: The distribution of the OSRVM A sound pressure level at the field point in the cab.

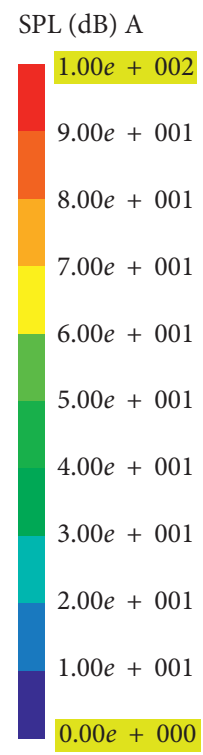

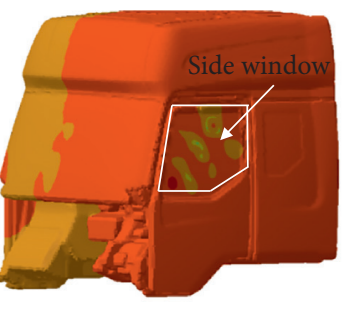

OSRVM A

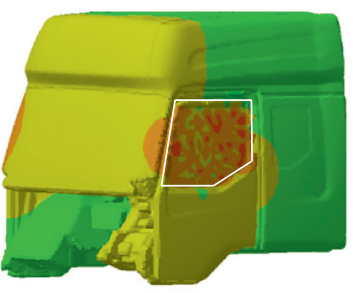

OSRVM A

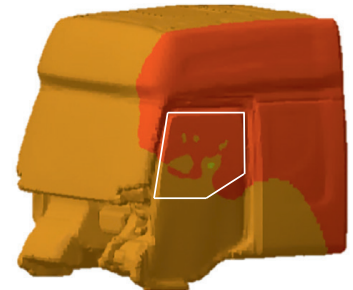

OSRVM B

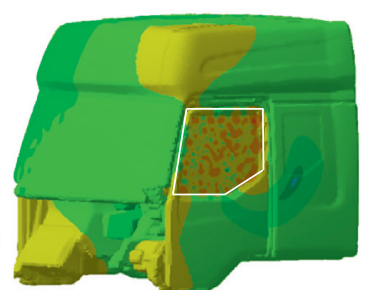

OSRVM B

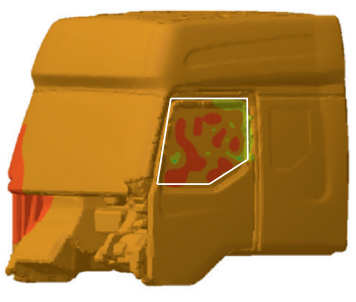

OSRVM C

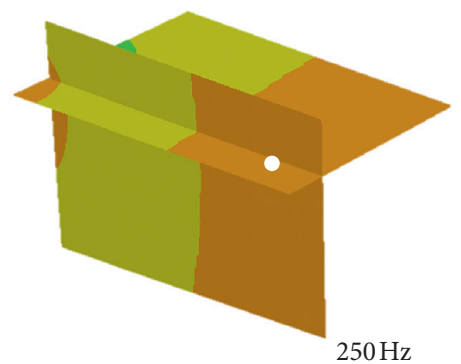

Three OSRVM mode and NO OSRVM in $500 \mathrm{~Hz}$

FIgURE 11: The distribution of the sound pressure level of four statuses in the cab cavity.

rear-view mirror than on its side. The SPL in the car changes at $500 \mathrm{~Hz}$ and gradually decreases.

Figure 13 summarizes the spectrum of the $1 / 3$ octave centre frequency at the driver's right ear for the three OSRVMs and for NO OSRVM. And the 1/3 octave was considered to be a frequency band division method that is more in line with the characteristics of the human ear. The rate of change corresponding to the $1 / 3$ octave centre frequency based on the vehicle speed is similar among the three OSRVM and NO OSRVM modes, where the maximum SPL of the exterior mirrors occurs at approximately $400 \mathrm{~Hz}$. Multiple peaks appear in the $20-2500 \mathrm{~Hz}$ band, which also confirms that the aerodynamic noise is broadband [12, 29, 30]. As the frequency increases, the SPL shows a downward trend, indicating that the aerodynamic noise energy is reflected primarily in the middle- and low-frequency bands. The amplitude of the noise is approximately $60 \mathrm{~dB}$, which easily fatigues the driver's auditory nerve and significantly affects the driving comfort. The SPL spectrum for OSRVM A is the largest and OSRVM C is the lowest (thus, optimal) at approximately $10 \mathrm{~dB}$ lower. This echoes the SPL cloud map for the vehicle interior. Similarly, the curve of the $1 / 3$ octave centre frequency SPL under NO OSRVM is significantly lower. 
SPL (dB) A

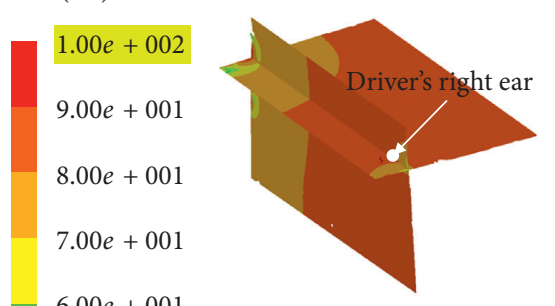

$6.00 e+001$

$5.00 e+001$

$4.00 e+001$

$3.00 e+001$

$2.00 e+001$

$1.00 e+001$

$0.00 e+000$
OSRVM A

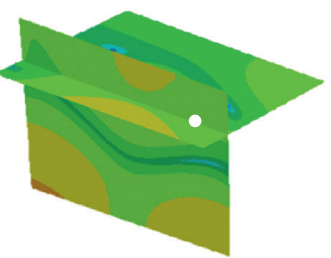

OSRVM A

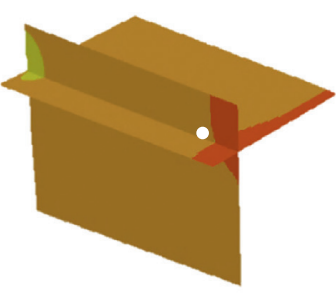

OSRVM B

Three OSRVM modes and NO OSRVM in $400 \mathrm{~Hz}$

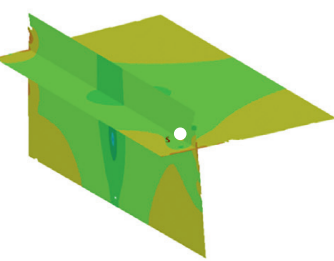

OSRVM B

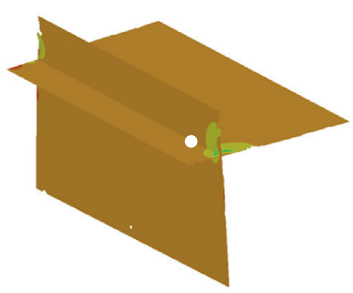

OSRVM C
On $400 \mathrm{~Hz}$

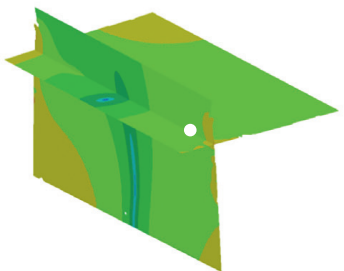

OSRVM C

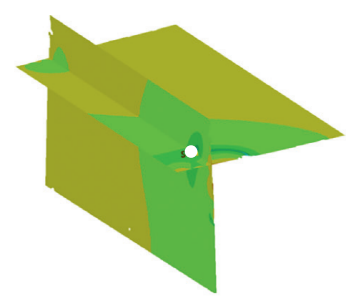

NO OSRVM

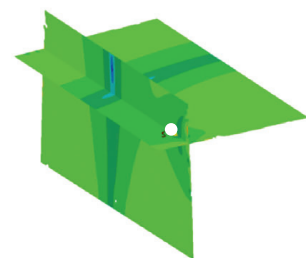

NO OSRVM

Three OSRVM modes and NO OSRVM in $500 \mathrm{~Hz}$

Figure 12: The distribution of the sound pressure level of four statuses at the field point in the cab.

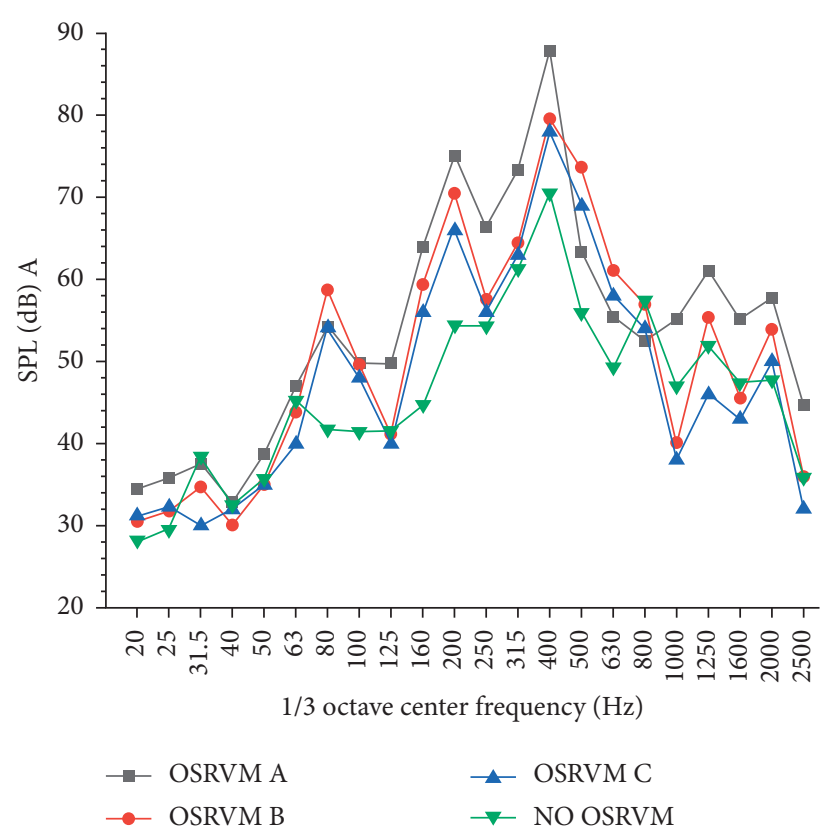

FIGURE 13: Comparison of sound pressure levels in the driver's left ear of three OSRVMs and NO OSRVM.

\section{Comparison between Simulation and Experiment}

Using acoustic wind tunnels for actual vehicle aerodynamic noise tests is expensive, which limits its practical use. Therefore, this paper performs road tests using an actual commercial at the high-speed flat asphalt road in the Xiangzhou state of Liuzhou. There were no other vehicles on the road surface, and the conditions were closer to the operations of high-speed CVs. The temperature was 24 degrees with a $1 \mathrm{~m} / \mathrm{s}$ breeze. The lab noise test equipment from LMS (Belgium) was used, and the Lab 17 A experimental analysis software system was integrated with a 16channel SCADAS portable data acquisition front end. Prepolarized microphones and preamplifiers from GRAS (Denmark, shown in Figure 14) were used, and the total sound pressure level at $100 \mathrm{mph}$ was measured using a handheld sound level meter. Figure 14(b) shows the test points and instruments for the road tests.

The sound pressure level (SPL) tests in the commercial measure the noise for the entire vehicle, and differences between the low-frequency band simulation and the test 


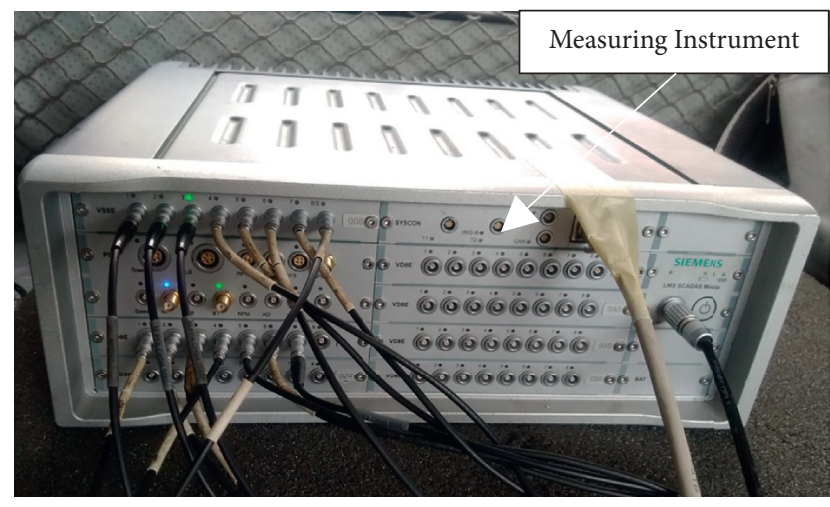

(a)

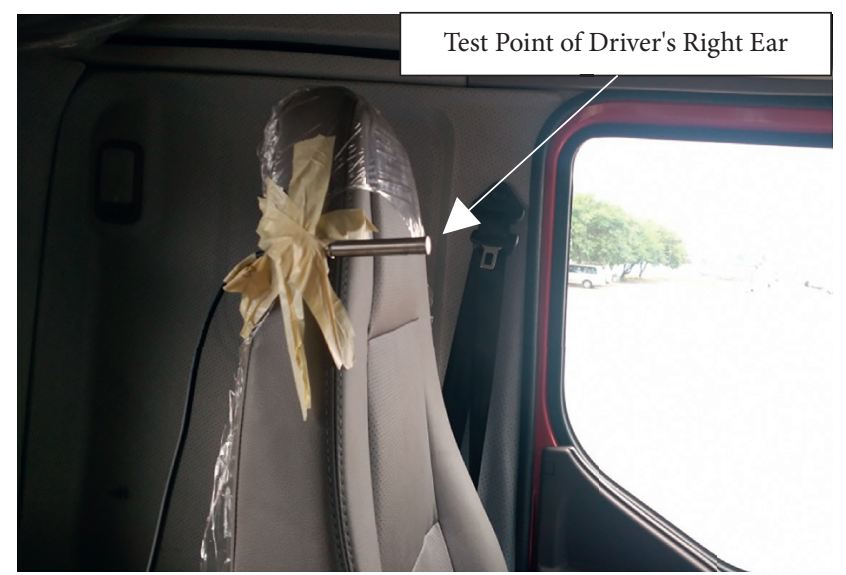

(b)

Figure 14: Noise testing equipment and arrangement of microphones. (a) Measuring instrument. (b) Test point of the driver's right ear.

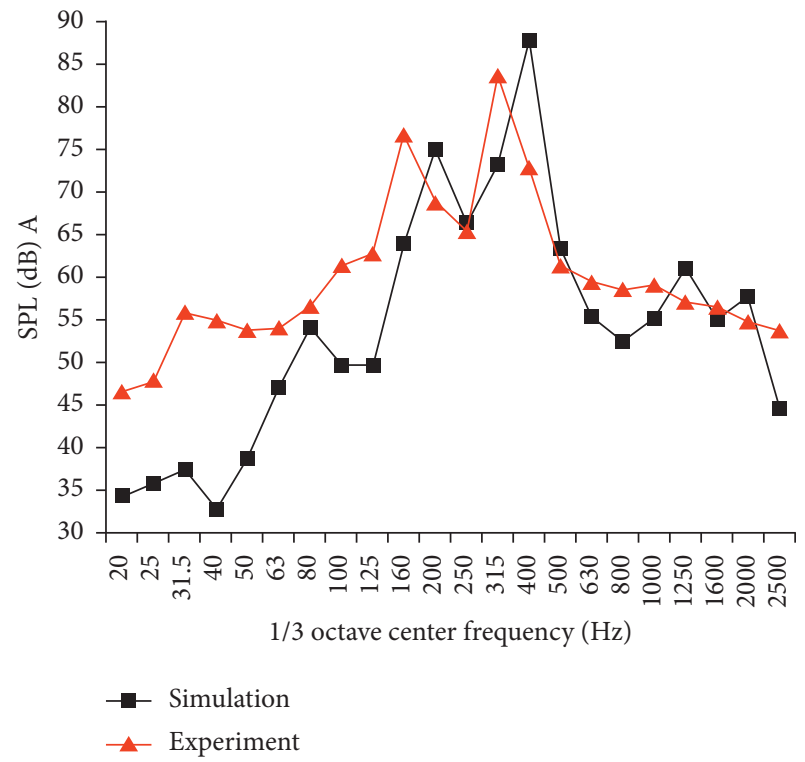

FIGURE 15: The comparison of the experiment and simulation on the $1 / 3$ octave centre frequency.

values are as much as $20 \mathrm{~dB}$, as shown in Figure 15. As the frequency increases, the differences between the simulations and experiments gradually decrease. In the middle-and high-frequency bands, they are in good agreement and show that the aerodynamic noise plays an important role. The simulated total SPL level is $85.56 \mathrm{~dB}$ and the experimental is $74.84 \mathrm{~dB}$, giving an error of nearly $11 \mathrm{~dB}$. The main causes of this error are the low-frequency structural vibration and noise caused by the engine and road surface excitation during the experiment, which amplifies the test values. During the simulation, the pressure pulsation of the window is considered, and the aerodynamic noise generated from the sun visor and other parts of the cab is ignored. In addition, the whistling sound caused by the hole on the surface of the car body and the aerodynamic noise caused by the separation of the airflow of the local step (such as the roof bump) are ignored.

\section{Conclusions}

A simulation methodology is presented in order to predict the interior acoustic field in the commercial vehicle cab generated by the outside rear-view mirror. The methodology relies on a hybrid aeroacoustic strategy, where the sound source field is obtained from unsteady surface pressure extracted on two side windows from an incompressible CFD solution by STAR-CCM+. The acoustic field is then solved with the surface dipole approach in the FEMAO framework of LMS Virtual.Lab. Based on hybrid simulation strategy analysis, the aerodynamic flow characteristics and interior acoustic field sound pressure levels for commercial vehicles with the trailer of the proposed three outside rear-view mirror models were compared. The comparative studies at none mirror status showed that the velocity/pressure profiles and sound pressure levels were dramatically improved for commercial vehicles with the trailer. And the following conclusions are made:

(1) It was showed that the proposed methodology is applicable to predict the aerodynamic noise inside the cab of commercial vehicles comparing the data of actual road test of the taxiing road. It is very beneficial to optimize the outside rear-view mirror based on the analysis of the wind drag and then to investigate the propagation of the aerodynamic noise into the cab interior.

(2) Various rear-view mirrors have different wind drags for commercial vehicles with the trailer. The greatest impact was approximately $1.2 \%$. The presence of the rear-view mirror can effectively reduce the wind drag for commercial vehicles with the trailer, comparing the data (4.9\%) from the state of commercial vehicles without the rear-view mirror.

(3) The guide cover of the rear-view mirror frame, the thickness of the rear-view mirror housing, and passivation affect different degrees of aerodynamic interior noise of the cab. Optimizing these parameters can reduce the aerodynamic noise inside the car as well as the wind drag. 


\section{Data Availability}

The data used to support the findings of this study are available from the corresponding author upon request.

\section{Conflicts of Interest}

The authors declare that there are no conflicts of interest regarding the publication of this paper.

\section{Acknowledgments}

This research was financially supported by the Promotion of Basic Ability of Young and Middle-aged Teachers in Guangxi Zhuang Autonomous Region (no. 2018KY0205), Guangxi Youth Science Foundation Project (2018GXNSFBA281012), Liuzhou Science Research and Planning Development Project (nos. 2019AD10203 and 2018AA20301), and Innovation-Driven Development Special Fund Project of GuangXi (GuiKe AA19182004).

\section{References}

[1] Y. Li, N. Kasaki, H. Tsunoda, T. Nakamura, and T. Nouzawa, "Evaluation of wind noise sources using experimental and computational methods," in Proceedings of the SAE Technical Paper Series, pp. 1-343, Detroit, MI, USA, April 2006.

[2] C. Peric, S. Watkins, and E. Lindqvist, "Wind turbulence effects on aerodynamic noise with relevance to road vehicle interior noise," Journal of Wind Engineering and Industrial Aerodynamics, vol. 69-71, pp. 423-435, 1997.

[3] K. Kucukcoskun, M. Eng, R. Boeykens et al., "Prediction of exterior noise generated by a side-mirror of an SUV," in Proceedings of the //INTER-NOISE and NOISE-CON congress and conference proceedings, pp. 3306-3316, Seoul, South Korea, August 2016.

[4] A. Schell and V. Cotoni, "Prediction of interior noise in a sedan due to exterior flow," SAE International Journal of Passenger Cars-Mechanical Systems, vol. 8, no. 3, pp. 10901096, 2015.

[5] Y.-J. Chu, Y.-S. Shin, and S.-Y. Lee, "Aerodynamic analysis and noise-reducing design of an outside rear view mirror," Applied Sciences, vol. 8, no. 4, p. 519, 2018.

[6] L. A. R. Mutnuri, S. Senthooran, R. Powell et al., "Computational process for wind noise evaluation of rear-view mirror design in cars," in Proceedings of the SAE Technical Paper Series, Detroit, MI, USA, April 2014.

[7] Z. Gu, Y. Zong, Z. Luo, Z. Yang, C. Jiang, and Z. Chen, "Numerical simulation of automobile side-window buffeting noise based on fluid-structure interaction," Applied Acoustics, vol. 90, pp. 126-137, 2015.

[8] K. Iida, K. Onda, A. Iida et al., "Prediction of aeroacoustical interior noise of a car, part-2 structural and acoustical analyses," in Proceedings of the SAE Technical Paper series, Detroit, MI, USA, April 2016.

[9] Y. Yamade, C. Kato, S. Yoshimura et al., "Prediction of aeroacoustical interior noise of a car, part-1 prediction of pressure fluctuations on external surfaces of a car," in Proceedings of the SAE Technical Paper Series, Detroit, MI, USA, April 2016.

[10] H. Zhu, W. Liu, T. Zhou et al., "Direct numerical simulation of the wake adjustment and hydrodynamic characteristics of a circular cylinder symmetrically attached with fin-shaped strips," Ocean Engineering, vol. 195, Article ID 106756, 2020.

[11] A. Baghlani, M. Khayat, S. M. Dehghan et al., "Free vibration analysis of FGM cylindrical shells surrounded by Pasternak elastic foundation in thermal environment considering fluidstructure interaction," Applied Mathematical Modelling, vol. 78, pp. 550-575, 2020.

[12] H. Zhu, G. Li, and J. Wang, "Flow-induced vibration of a circular cylinder with splitter plates placed upstream and downstream individually and simultaneously," Applied Ocean Research, vol. 97, Article ID 102084, 2020.

[13] H. Zhu, J. Yao, Y. Ma, H. Zhao, and Y. Tang, "Simultaneous CFD evaluation of VIV suppression using smaller control cylinders," Journal of Fluids and Structures, vol. 57, pp. 66-80, 2015.

[14] K. Yang, J. Wang, D. Yurchenko et al., "A double-beam piezomagneto-elastic wind energy harvester for improving the galloping-based energy harvesting," Applied Physics Letters, vol. 115, no. 19, 2019.

[15] J. Wang, S. Zhou, Z. Zhang, and D. Yurchenko, "High-performance piezoelectric wind energy harvester with Y-shaped attachments," Energy Conversion and Management, vol. 181, pp. 645-652, 2019.

[16] N. Murad, J. Naser, F. Alam, and S. Watkins, "Computational fluid dynamics study of vehicle A-pillar aero-acoustics," Applied Acoustics, vol. 74, no. 6, pp. 882-896, 2013.

[17] P. Spalart, "Trends in turbulence treatments," in Proceedings of the Fluids 2000 Conference and Exhibit, p. 2306, Denver, CO, USA, June 2000.

[18] M. Strelets, "Detached eddy simulation of massively separated flows," in Proceedings of the 39th Aerospace sciences meeting and exhibit, p. 879, Reno, NV, USA, January 2001.

[19] W.-S. Fu, Y.-C. Lai, and C.-G. Li, "Estimation of turbulent natural convection in horizontal parallel plates by the $\mathrm{Q}$ criterion," International Communications in Heat and Mass Transfer, vol. 45, pp. 41-46, 2013.

[20] J.-H. Kim and Y. O. Han, "Experimental investigation of wake structure around an external rear view mirror of a passenger car," Journal of Wind Engineering and Industrial Aerodynamics, vol. 99, no. 12, pp. 1197-1206, 2011.

[21] B. Lokhande, S. Sovani, and J. Xu, "Computational aeroacoustic analysis of a generic side view mirror," in Proceedings of the SAE Technical Paper Series, pp. 2175-2184, Detroit, MI, USA, May 2003.

[22] J. E. Fowcs Williams and D. L. Hawkings, "Sound generation by turbulence and surfaces in arbitrary motion," Philosophical Transactions of the Royal Society of London. Series A, Mathematical and Physical Sciences, vol. 264, no. 1151, pp. 321-342, 1969.

[23] Y. Kato, "Numerical simulations of aeroacoustic fields around automobile rear-view mirrors," SAE International Journal of Passenger Cars-Mechanical Systems, vol. 5, no. 1, pp. 567-579, 2012.

[24] G. Balasubramanian, L. A. R. Mutnuri, Z. Sugiyama et al., “A computational process for early stage assessment of automotive buffeting and wind noise," SAE International Journal of Passenger Cars-Mechanical Systems, vol. 6, pp. 1231-1238, 2013.

[25] H. Zhu and J. Yao, "Numerical evaluation of passive control of VIV by small control rods," Applied Ocean Research, vol. 51, pp. 93-116, 2015.

[26] J. Wang, S. Zhou, Z. Zhang, and D. Yurchenko, "High-performance piezoelectric wind energy harvester with Y-shaped 
attachments," Energy Conversion and Management, vol. 181, pp. 645-652, 2019.

[27] G. Hu, J. Wang, Z. Su et al., "Performance evaluation of twin piezoelectric wind energy harvesters under mutual interference," Applied Physics Letters, vol. 115, no. 7, 2019.

[28] J. Wang, L. Tang, L. Zhao, and Z. Zhang, "Efficiency investigation on energy harvesting from airflows in HVAC system based on galloping of isosceles triangle sectioned bluff bodies," Energy, vol. 172, pp. 1066-1078, 2019.

[29] Q. Zhu, S. K. Stoter, M. Heisel et al., "Reducing wind-induced vibrations of road sign structures through aerodynamic modifications: a computational pilot study for a practical example," Journal of Wind Engineering and Industrial Aerodynamics, vol. 199, Article ID 104132, 2020.

[30] J. Wang, S. Gu, C. Zhang et al., "Hybrid wind energy scavenging by coupling vortex-induced vibrations and galloping," Energy Conversion and Management, vol. 213, Article ID 112835,2020 